\title{
High frequency oscillations in low-dimensional conductors and semiconductor superlattices induced by current in stack direction
}

\author{
S.N. Artemenko, S.V. Remizov \\ Institute of Radio-engineering and Electronics of Russian Academy of Sciences, 11-7 Mokhovaya str., Moscow 125009, Russia
}

(Dated: January 2, 2019)

\begin{abstract}
Narrow energy band of the electronic spectrum in some direction in low-dimensional crystals may lead to a negative differential conductance and N-shaped I-V curve that results in an instability of the uniform stationary state. Well-known stable solution for such a system is a state with electric field domain. We have found a uniform stable solution in the region of negative differential conductance. This solution describes uniform high frequency voltage oscillations. Frequency of the oscillation is determined by antenna properties of the system. The results are applicable also to semiconductor superlattices.

PACS numbers: 73.21.Cd, 73.21.Ac, 72.80.-r
\end{abstract}

\section{INTRODUCTION}

There is a number of materials with narrow energy band of the electronic spectrum for electrons moving in certain directions. The narrow band may result either from low-dimensional structure of the crystal or from artificially made periodic superstructure like semiconductor superlattices. Typical examples of low-dimensional crystals are layered materials such as high-Tc superconductors, transition metal dichalcogenides like $\mathrm{TaS}_{2}, \mathrm{NbSe}_{2}$ and others 1 . Such materials can be treated as a stack of conducting layers of atomic thickness, separated by insulating ones ${ }^{2}$. Narrow electronic bands are typical also for quasi-one-dimensional conductors in directions perpendicular to conducting chains. For example, the linear chain conductor $\mathrm{NbSe}_{3}$ is a strongly anisotropic crystal with the anisotropy of conductivity $\sigma_{b} / \sigma_{c} \sim 10$ in (b-c) plane, and $\sigma_{b} / \sigma_{a^{*}} \sim 10^{4}$ in (a-b) plane at low temperatures ${ }^{3}$. Such a strong anisotropy reflects weak inter-chain coupling and narrow electronic bands for electron motion across conducting chains.

This feature of energy spectrum should lead to similarities of the effects observed in low-dimensional materials and

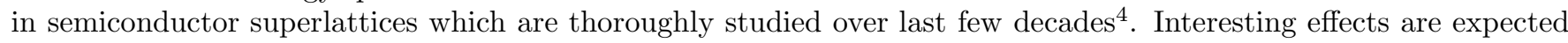
if electron motion approaches a regime close to the Bloch oscillations, when applied voltage is high enough and an electron has enough time to perform several oscillations within the same mini-band until it is scattered, $i$. $e$., when period of the Bloch oscillations is smaller then the momentum scattering time, $\tau_{p}$. In such a regime DC current decreases with DC voltage increasing, resulting in a region of negative differential conductance (NDC). This result was predicted theoretically for superlattices by Esaki and $\mathrm{Tsu}^{5}{ }^{5}$, and later was observed repeatedly. Similar conclusions were made for layered conductors ${ }^{6}$. However, to our knowledge, there is no direct experimental confirmation of this effect in low-dimensional conductors. A possible reason for this is that the crystals were not perfect enough to ensure the band motion of electrons in directions of low conductivity in low-dimensional materials. However, the strong conductivity maximum at zero bias voltage observed recently in $\mathrm{NbSe}_{3}$ by Latyshev et $a l_{\underline{\underline{7}} \underline{\underline{7}}}$ argues in favor of possibility of the NDC in such materials.

An important corollary of the NDC is an instability of uniform stationary state in the NDC region which, in principle, may result in generation of high frequency oscillations when the DC voltage gets into the NDC region. The boundary of the NDC region in the static I-V curve is determined by the voltage related to the momentum scattering rate $\tau_{p}^{-1}$, and the upper frequency limit for the NDC is equal to $\tau_{p}^{-1}$ as well. The value of $\tau_{p}^{-1}$ in many materials falls into the $\mathrm{THz}$ region ${ }^{7}, \frac{8}{,}$, so one can expect microwave generation in this frequency range. However, there is a number of obstacles preventing generation of high frequency oscillations. The primary one is formation of nonuniform electric field distribution (field domains) which impedes high frequency oscillations due to relatively low domain velocity ${ }^{4}$. A number of techniques was suggested to overcome this difficulty in superlattices, including application to a sample additional large $\mathrm{AC}^{9}$ and using of pulse voltage $\stackrel{10}{\underline{1}}$, or formation of superlattices with minibands having non-sinusoidal dispersion law 11 , etc.

In this work we study theoretically a low-dimensional conductor with current flowing in the direction of low conductivity when applied DC voltage falls into the NDC region, and find a uniform oscillatory solution in case when no external time-dependent influence is imposed. This solution is possible because of interaction of a sample with an environment which we describe by means of an effective antenna impedance. We found conditions for existence of stable oscillations: the antenna reactance on main oscillation frequency must have the sign corresponding to an inductance, in this case the sample and the effective antenna form an effective oscillatory circuit. The frequency of the oscillations is determined by the antenna reactance and by material properties of the sample. Oscillation amplitude depends on applied voltage and vanishes at the boundaries of the NDC region. 
The paper is organized as follows: in Sec.[1] we derive general expression for the current across the layers. In Sec. III we study in details the uniform current flow through the sample, derive equations of motion for time-dependent voltage, and find a solution describing oscillations. We show first the possibility of coherent voltage oscillations using oversimplified but physically transparent model, and then bring strict solution in the form of harmonic series. In Sec. IV we examine response of the system to a small external time-dependent perturbation, and in Sec. $\nabla$ we demonstrate that solution describing coherent voltage oscillations in the sample is stable with respect to small fluctuations. Finally, in Sec. VD we discuss conditions for experimental realization of the oscillatory regime found in preceding sections and make conclusions.

\section{MAIN EQUATIONS}

As it was mentioned in the Introduction, electron motion across the layers of perfect enough layered conductor, or between the conducting chains in quasi-one-dimensional conductor can be considered as a motion in narrow conducting band that should have specific features typical for electron motion within the minibands in semiconductor superlattices. In the theory of miniband transport there are two main limiting cases. If electron wave functions in adjacent layers are strongly overlapped then an electron has enough time to perform several tunneling processes until it is scattered. This quasi-classical case of miniband conduction can be well described by means of Bolzman equation which holds when $\tau_{p}^{-1} \ll \Delta$, where $\Delta$ is the miniband width. If the interlayer hopping integral is small then the sample may be treated as a stack of weakly coupled quantum wells, hence, tunneling processes in adjacent junctions are practically independent, and purely quantum case of sequential tunneling is fulfilled. An amazing result of supperlattice theory is that in both these limits expressions describing the current as functional of voltage are the same ${ }^{4}$. Below we show how the similar expression can be derived for a low-dimensional conductors. For definiteness we will speak about a layered conductor with voltage applied in the stack direction.

For brevity we use units with $e=\hbar=1$. To investigate non-equilibrium transport we use the Keldysh technique for Green functions ${ }^{12}$. Current density between layers $n$ and $n+1$ may be expressed in terms of the non-diagonal component of Green's function both on temporal indices and layer numbers: $j_{n, n+1}(\tau)=$ $i e t_{\perp} \hbar^{-1}\left(G_{n, n+1}^{K}(\tau, \tau)-G_{n+1, n}^{K}(\tau, \tau)\right)$,

$$
\hat{G}_{n, m}=\left(\begin{array}{cc}
G_{n, m}^{R} & G_{n, m}^{K} \\
0 & G_{n, m}^{A}
\end{array}\right)
$$

where $t_{\perp}$ is the hopping integral for electron tunneling between the adjacent layers, which is supposed to be independent on layer number and electron momentum, and $G^{R(A)}$ is the retarded (advanced) Green function, $\tau$ is the dimensionless time, which is mesured in units of $\tau_{p}$. For convenience we introduce other dimensionless units: frequencies and energies we mesure in terms of $\tau_{p}^{-1}$.

Usually, the hopping integral, $t_{\perp}$, is small and it is possible to construct a perturbation theory, which allows us to express non-diagonal on layer number Green functions in terms of diagonal ones. In the first approximation on $t_{\perp}$ it reads $\hat{G}_{n, n+1}\left(\tau, \tau^{\prime}\right)=t_{\perp} \int \hat{G}_{n, n}\left(\tau, \tau^{\prime \prime}\right) \hat{G}_{n+1, n+1}\left(\tau^{\prime \prime}, \tau^{\prime}\right) d \tau^{\prime \prime}$, and, hence, we get well known expression for the current between the adjacent layers $\frac{13}{}$.

$$
j_{n, n+1}(\tau)=i t_{\perp}^{2} \int G_{n, n}^{R}\left(\tau, \tau^{\prime}\right) G_{n+1, n+1}^{K}\left(\tau^{\prime}, \tau\right)+G_{n, n}^{K}\left(\tau, \tau^{\prime}\right) G_{n+1, n+1}^{A}\left(\tau^{\prime}, \tau\right) d \tau^{\prime} .
$$

We consider the case when electrons inside the layer are in in the equilibrium while layers are not in the equilibrium with each other. A difference between $j_{n-1, n}$ and $j_{n, n+1}$ leads to deviation $\delta \mu_{n}$ of the chemical potential in layer $n$ from its equilibrium value, $\mu$. In this case in each layer the diagonal Green function with respect to layer number, $G_{n, n}^{K}$, can be related to the retarded and advanced Green functions by the expression valid for the equilibrium case: $G_{n, n}^{K}(\omega)=\left(G_{n, n}^{R}(\omega)-G_{n, n}^{A}(\omega)\right) \tanh \left(\omega-\delta \mu_{n}\right) / 2 T$, here we take into account temporal uniformity, so that $G_{n, n}^{R(A)}\left(\omega, \omega^{\prime}\right)=G_{n, n}^{R(A)}(\omega) \delta\left(\omega-\omega^{\prime}\right)$. The transition to temporal representation we perform according to

$$
G_{n, n}^{R(A)}\left(\tau^{\prime}, \tau^{\prime \prime}\right)=\int e^{i \omega^{\prime} \tau^{\prime}-i \omega^{\prime \prime} \tau^{\prime \prime}} G_{n, n}^{R(A)}\left(\omega^{\prime}, \omega^{\prime \prime}\right) e^{\int_{\tau^{\prime}}^{\tau^{\prime \prime}} \Phi_{n}(\tau) d \tau} d \omega^{\prime} d \omega^{\prime \prime}
$$

here $\Phi_{n}(\tau)$ is time-dependent scalar electric potential of corresponding layer. To give proper account to scattering, we use the standard expression for Green functions with non-zero momentum relaxation rate $G_{n, n}^{R(A)}(\omega)=\left(\omega-\xi_{p} \pm i / 2\right)^{-1}$, here " \pm " is related to the retarded (advanced) Green function, respectively, $\xi_{p}$ is the electron energy, counted from the Fermi energy, $p$ is the component of electron momentum parallel to the layers. 
We assume for simplicity that $\tau_{p}$ and $t_{\perp}$ do not depend on electron momentum. We concentrate on the case of the same voltage on all junctions, taking the non-uniformity into account only in Sec. $\nabla$ where we study the stability of the uniform solution in respect to small perturbations. So we assume that deviations, $\delta \mu_{n}$, of the chemical potential from the equilibrium value are small.

Finally, the expression for the tunneling current between adjacent layers reads

$$
\begin{aligned}
j_{n}^{(t)}(\tau)= & \frac{\sigma_{0}}{s}\left(\int_{0}^{\infty} d \tau_{1} e^{-\tau_{1}} \sin \int_{0}^{\tau_{1}}\left(V_{n}(\tau)-V_{n}\left(\tau-\tau_{2}\right)\right) d \tau_{2}-\right. \\
& \left.\left(\delta \mu_{n+1}-\delta \mu_{n}\right) \int_{0}^{\infty} d \tau_{1} e^{-\tau_{1}} \cos \int_{0}^{\tau_{1}}\left(V_{n}(\tau)-V_{n}\left(\tau-\tau_{2}\right)\right) d \tau_{2}\right),
\end{aligned}
$$

where $s$ is the lattice period in the direction of the current. The second item in square brackets in Eq. (1D) describes the diffusion current, $V_{n}(\tau)$ is the voltage between layers $n+1$ and $n, \sigma_{0}=m e^{2} t_{\perp}^{2} \tau_{p} / \hbar$ is conductivity at zero bias and frequency, and $s$ is the period of the structure.

Consider now limiting forms and simplifications of equation (11) for the current. We represent the voltage across the layer as a sum $V(\tau)=v_{0}+v(\tau)$, where $v_{0}$ is the average voltage (here we drop index $n$ ). When the second time-dependent term is small Eq. (11) can be expanded in series with respect to $v(\tau)$, and we obtain for the current:

$$
j^{(t)}(\tau)=j_{0}+\frac{1}{s}\left(\sigma_{d} v(\tau)+\frac{1}{2} \sigma_{d}^{\prime} v(\tau)^{2}+\frac{1}{6} \sigma_{d}^{\prime \prime} v(\tau)^{3}\right)+\left(c_{t}+c_{t}^{\prime} v(\tau)\right) \partial_{\tau} v(\tau)
$$

Here we introduce the DC part of the current, $j_{0}(v)=\sigma v_{0} / s$ where $\sigma=\sigma_{0} /\left(1+v_{0}^{2}\right)$. Further, $\sigma_{d} \equiv j_{0}^{\prime} \equiv d j_{0} / d v_{0}=$ $\sigma_{0}\left(1-v_{0}^{2}\right) /\left(1+v_{0}^{2}\right)^{2}$ is the differential conductance, and $c_{t}=\left(3 v_{0}^{2}-1\right) / s\left(1+v_{0}^{2}\right)^{3}$ is the specific tunneling capacity of the sample. The apostrophe denotes derivative on voltage $v_{0}$.

As an illustration, consider, first, the simplest case when the applied voltage is time-independent, $v(\tau)=0$. Then Eq. (2) results in well-known form ${ }^{5}: j=j_{0} \sim v_{0}\left(1+v_{0}^{2}\right)^{-1}$. It follows from this expression that the differential conductance becomes negative when $v_{0}>1$.

In case of small harmonic voltage, $v(\tau)=v_{1} e^{i \omega \tau}$ the expression for the current in the first approximation in $v(\tau)$ reads

$$
j^{(t)}(\tau)=j_{0}+v_{1} \frac{i \omega\left(v_{0}^{2}-1-i \omega\right) \sigma_{0}}{s\left(v_{0}^{2}-(\omega-i)^{2}\right)\left(v_{0}^{2}+1\right)} e^{i \omega \tau} .
$$

From this expression it follows that at high frequencies, $\omega \gtrsim 1$, the differential conductivity becomes positive even when the DC bias is in the NDC region.

\section{COHERENT VOLTAGE OSCILLATIONS}

In this section we derive equation of motion for the voltage and study dynamics of the system when currents in all junctions are identical. In addition, we neglect voltage fluctuations here, therefore, we do not consider non-equilibrium deviations of chemical potential in different layers and, hence, the diffusion current.

In addition to the tunneling current (10) there are other contributions to the total current. First, we must take into account the displacement current

$$
j^{(d i s p l)}=c_{0} \frac{\partial V(\tau)}{\partial \tau},
$$

where we use notation for the specific capacity of the sample $c_{0}=1 / 4 \pi s$.

We must take into account also leakage currents, contributions due to incoherent tunneling, and other processes which are not related to coherent tunneling. To allow for these effects phenomenologically we add an extra ohmic term to the current:

$$
j^{(o h m)}(\tau)=\sigma_{1} \frac{V(\tau)}{s} .
$$

For the I-V curve to have the NDC region conductivity $\sigma_{1}$ must be small enough:

$$
\sigma_{1}<\sigma_{0} / 8 \text {. }
$$


To determine two independent variables, the current and the applied voltage, an additional equation relating the applied voltage to the current in the external to the sample circuit is required. The equation for current balance which determines the voltage dynamics reads

$$
j^{(t)}+j^{(o h m)}+j^{(d i s p l)}+j^{(e x t)}=0
$$

Here $j^{(e x t)}=j_{A}+j_{S}$ is the current in the circuit external to the sample. We consider this circuit as that consisting of a power supply and environment. Coupling of the sample to the environment we describe by means of an effective antenna with the impedance $Z_{A}$. The equivalent scheme for the system under consideration is shown in Fig. 1. Since typical frequencies of the problem is high, we assume that $\mathrm{AC}$ current does not flow through power supply, so that antenna current $j_{A}$ is $\mathrm{AC}$ and power supply current $j_{S}$ is DC.

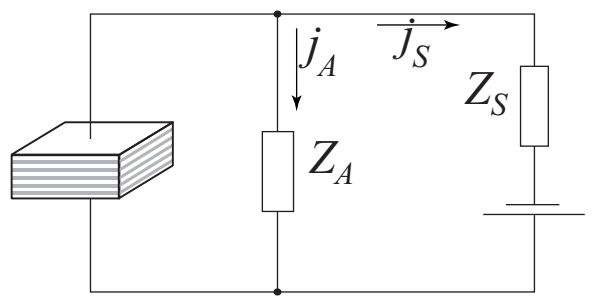

FIG. 1: Equivalent circuit of the system. $Z_{A}$ and $Z_{S}$ are impedances of the antenna and of the power supply, $j, j_{A}$, and $j_{S}$ are the currents flowing through the sample, antenna, and power supply, respectively. Current in the external circuit $j^{(e x t)}=j_{S}+j_{A}$. We assume on frequencies of the problem $\left|Z_{A}\right| \ll\left|Z_{S}\right|$ and on zero frequency $\left|Z_{A}\right| \gg\left|Z_{S}\right|$ so that $j_{A}$ is $\mathrm{AC}$ and $j_{S}$ is DC.

Sample dimensions are assumed to be small with respect to radiation wavelength and to the skin-effect length. This allows us to neglect spatial non-uniformity.

The resulting equation describing dynamics of the voltage oscillations has rather complicated form of the integrodifferential equation. In order to find an analytical solution we consider the limit in which the amplitude of the voltage oscillations is small in comparison to the DC voltage, $v(\tau) \ll v_{0}$. This regime occurs near the boundary of the NDC region or in the case when the NDC region is small.

\section{A. Simple model}

It is instructive to examine, first, an oversimplified, but physically transparent model, based on assumption that in the frequency range under consideration the effective antenna can be described by the admittance with purely inductive reactive part: $j_{A}(v) \rightarrow \frac{N}{L S} \int v(\tau) d \tau$. Here $L$ is the antenna inductance, and factor $N$ is number of layers in the sample, so that the total voltage across the sample is equal to $N v(\tau)$. The real part of the antenna admittance leads to redefinition of the conductivity $\sigma_{1}$ only, therefore, we drop it here. For convenience we introduce here a new variable related to the uniform alternating electric field in the sample as $\dot{\theta}=v(\tau) / s$, and assume that the NDC region is small, so that contribution of the second harmonic into the friction terms is negligible (see the next subsection). Eq. (77) then reads:

$$
\ddot{\theta}+\omega_{0}^{2} \theta+\frac{1}{c_{s} s}\left(\delta \dot{\theta}+\frac{\sigma_{d}^{\prime} \dot{\theta}^{2}}{2}+\frac{\sigma_{d}^{\prime \prime} \dot{\theta}^{3}}{6}-j+\sigma_{1} \frac{v_{0}}{s}\right)=0 .
$$

Here $j$ is DC current flowing through the sample, $\omega_{0}=\sqrt{N / L S c_{s}}, S$ is the sample area in the plane perpendicular to the current direction, and $N$ is the number of junctions, and we have introduced the total specific capacity of the sample, $c_{s}=c_{0}+c_{t}$, and supercriticality, $\delta=\sigma_{d}+\sigma_{1}$, which changes its sign from "-" to "+" across zero when the $\mathrm{DC}$ voltage leaves the instability region of the $\mathrm{I}-\mathrm{V}$ curve.

Equation for oscillations (8) can be treated as a mechanical equation of motion. In this equation $\theta$ plays a role of a coordinate, the last term in the left-hand side of Eq. (8) corresponds to a time-dependent alternating friction. In the regime of stable oscillations the friction averaged over the period must be equal to zero, and its momentary value can be considered perturbatively. So, in the first approximation we can neglect the friction term, $\ddot{\theta}^{(1)}+\omega_{0}^{2} \theta^{(1)}=0$. Since the phase of the solution is arbitrary, for definiteness, we choose it in the form $\dot{\theta}^{(1)}=E_{1} \sin \omega_{0} \tau$. $E_{1}$ is an amplitude of electric field oscillations on main frequency, so voltage oscillations amplitude on this frequency is $E_{1} s$. To ensure 
that the solution does not increase with time the friction term in (8) must not contribute at the oscillation frequency $\omega_{0}$. This condition determines the amplitude of the oscillations

$$
E_{1}=\sqrt{-\frac{8 \delta}{\sigma_{d}^{\prime \prime}}}
$$

Guided by mechanical analogy mentioned above, we multiply friction term in Eq. (8) by $\theta_{1}$ and integrate over the period and demand that the work of the friction force over the oscillation period is zero. The condition of vanishing of the average friction determines the I-V curve:

$$
j\left(v_{0}\right)=j_{\text {stat }}-2 \frac{\sigma_{d}^{\prime} \delta}{\sigma_{d}^{\prime \prime}} .
$$

This dynamic I-V curve intersect the static one $j_{\text {stat }}=\left(\sigma_{1}+\sigma_{0} /\left(1+v_{0}^{2}\right)\right) v_{0}$ at three points: at the boundaries of the NDC region and at $v_{0}=\sqrt{3}$ (see fig. 2).

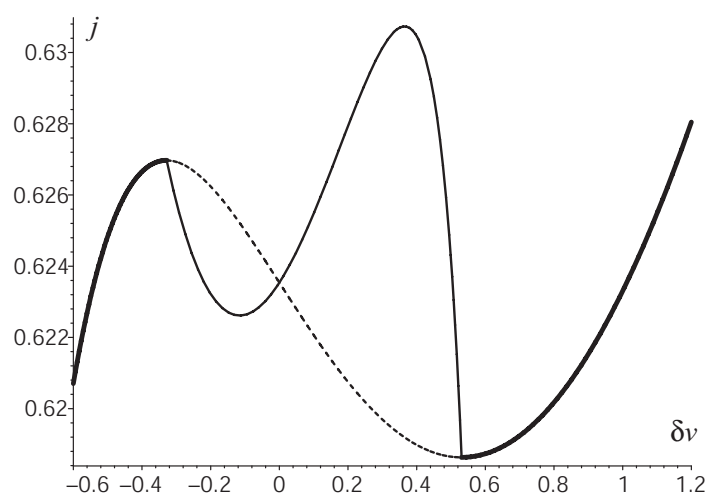

FIG. 2: Dynamic I-V curve is shown with the bold line. The dashed line describes the static I-V curve. Current is measured in dimensionless units, voltage $\delta v$ is counted from $v_{0}=\sqrt{3}$ corresponding to the middle of a small NDC region. The I-V curve is presented for $\sigma_{1}=0.11$.

\section{B. Solution in the form of harmonic series}

In this subsection we consider a more general case and determine conditions for existence of the oscillatory solution. We seek the solution of Eq. (77) for $v(\tau)$ in the form of harmonic series

$$
v(\tau)=\sum_{n=1}^{\infty}\left(v_{s, n} \sin \left(n \omega_{0} \tau\right)+v_{c, n} \cos \left(n \omega_{0} \tau\right)\right),
$$

In the most general case the antenna current reads

$$
j_{A}=\frac{N}{s} \sum_{n=1}^{\infty}\left(\sigma_{A n}^{R} v_{s, n}+\sigma_{A n}^{I} v_{c, n}\right) \sin n \omega \tau+\left(\sigma_{A n}^{R} v_{c, n}-\sigma_{A n}^{I} v_{s, n}\right) \cos n \omega \tau .
$$

Here $\sigma_{A n}^{R}=R_{n} s /\left(R_{n}^{2}+X_{n}^{2}\right) S, \sigma_{A n}^{I}=X_{n} s /\left(R_{n}^{2}+X_{n}^{2}\right) S$, where $R_{n}$ and $X_{n}$ are resistance and reactance of the antenna at frequency $n \omega_{0}$ respectively.

Solution (11) has an arbitrary phase which can be determined by initial conditions. We may fix this phase without any restriction assuming that $v_{c, 1}=0$. The supercriticality parameter, $\delta_{n}=N \sigma_{A n}^{R}+\sigma_{1}+\sigma_{d}$, we assume to be small at the main oscillation frequency: $\left|\delta_{1}\right| \ll 1$. As it will be shown later, amplitudes of the $n$-th harmonic are proportional to $\delta_{1}^{n / 2}$, hence, it is possible to neglect the terms of the order higher than the third one in the small parameter $\sqrt{\delta_{1}}$. So inserting Eq. (11) into the expression for the current (10), and using equation for the current balance (7), we obtain 
a system of linear equations for $v_{s, 1}^{2}, v_{s, 2}$ and $v_{c, 2}$ :

$$
\left\{\begin{aligned}
-\sigma_{d}^{\prime \prime} v_{s, 1}^{2}+4 \omega c_{t}^{\prime} s v_{s, 2}+4 \sigma_{d}^{\prime} v_{c, 2} & =8 \delta_{1} s \\
\sigma_{d}^{\prime} v_{s, 2}-c_{t}^{\prime} s \omega v_{c, 2} & =2 N \sigma_{A 1}^{I}-2 c_{s} s \omega \\
\omega c_{t}^{\prime} s v_{s, 1}^{2}+2 \delta_{2} v_{s, 2}-2 \sigma_{2} v_{c, 2} & =0 \\
\sigma_{d}^{\prime} v_{s, 1}^{2}-4 \sigma_{2} v_{s, 2}-4 \delta_{2} v_{c, 2} & =0 .
\end{aligned}\right.
$$

Here $\sigma_{2}=2 c_{s} \omega-N \sigma_{A 2}^{I}$. Physically reasonable oscillation frequency is determined by the second equation of the system (12):

$$
\omega \simeq \frac{N \sigma_{A 1}^{I}}{c_{s} s}
$$

Here we take into account that the contribution of the second harmonic is of the second order in $\sqrt{\delta_{1}}$, so that lefthand sinde of this equation should be considered as higher order correction and, hence, should be neglected in main approximation. For oscillation amplitudes we find

$$
\begin{gathered}
v_{s, 1}^{2}=-8 \delta_{1} \frac{\sigma_{2}^{2}+\delta_{2}^{2}}{\mathcal{D}} \\
v_{s, 2}=2 \delta_{1} \frac{2 \omega \delta_{2} c_{t}^{\prime} s-\sigma_{d}^{\prime} \sigma_{2}}{\mathcal{D}} \\
v_{c, 2}=-2 \delta_{1} \frac{2 \omega c_{t}^{\prime} s \sigma_{2}+\delta_{2} \sigma_{d}^{\prime}}{\mathcal{D}}, \\
\mathcal{D}=-3 \sigma_{d}^{\prime} \omega c_{t}^{\prime} s \sigma_{2}+\left(2 \omega^{2}{c_{t}^{\prime}}^{2} s^{2}-{\sigma_{d}^{\prime}}^{2}\right) \delta_{2}+\left(\sigma_{2}^{2}+\delta_{2}^{2}\right) \sigma_{d}^{\prime \prime} .
\end{gathered}
$$

This expressions have a transparent physical meaning: if $\mathcal{D}>0$ the existence of the oscillatory regime is determined by the sign of the supercriticality, $\delta_{1}$. Oscillations are possible, (i.e., the amplitude of the first harmonic is real) only inside of the NDC region.

Now we show that $\mathcal{D}$ is positive under conditions of our assumption that harmonics drop rapidly. If the NDC region is small then the voltage is close to the center of the NDC region, $\left|v_{0}-\sqrt{3}\right| \ll 1$. Then $\sigma_{d}^{\prime} \propto v_{0}^{2}-3$ is small and $\mathcal{D}$ is positive. If the NDC region is large then our approach is valid near the boundaries of the NDC region. Near the low-voltage boundary $v_{0} \sim 1$. In this case $\sigma_{d}, c_{t}^{\prime} \rightarrow 0$ as $v_{0}-1$ and $\sigma_{d}^{\prime} \simeq 1 / 2, \sigma_{d}^{\prime \prime} \simeq 3 / 2$. So if $\delta_{2}$ and/or $\gamma_{2}$ are large enough, then $\mathcal{D}>0$. The high-voltage boundary of the NDC region corresponds to $v \gg 1$. In this case $c_{t}^{\prime}, \sigma_{d}^{\prime} \sim v_{0}^{-3}$, $\sigma_{d}^{\prime \prime} \sim v_{0}^{-4}$, and two first terms in (17) are small in comparison to the last one and, hence, $\mathcal{D}>0$. In other words, in this case the influence of second harmonic on the amplitude of the first one is small, so that $v_{s, 1} \simeq \sqrt{-8 \delta_{1} / \sigma_{d}^{\prime \prime}}$, as it was found in the previous subsection.

At the end of this chapter we consider the amplitudes of the higher harmonics. Acting perturbatively, we can express the amplitude of the third harmonic in terms of amplitudes of the first and the second harmonics considering the last ones as an "external force":

$$
\begin{gathered}
v_{c, 3}=-v_{s, 1} \frac{-\sigma_{A 3}^{I} \sigma_{d}^{\prime \prime} v_{s, 1}^{2}+12 \sigma_{d}^{\prime}\left(\sigma_{A 3}^{I} v_{c, 2}-\delta_{3} v_{s, 2}\right)}{24\left(\delta_{3}^{2}+\sigma_{A 3}^{I}{ }^{2}\right)} \sim \delta_{1}^{3 / 2}, \\
v_{s, 3}=-v_{s, 1} \frac{12 \sigma_{d}^{\prime}\left(\sigma_{A 3}^{I} v_{s, 2}+\delta_{3} v_{c, 2}\right)-\delta_{3} \sigma_{d}^{\prime \prime} v_{s, 1}^{2}}{24\left(\delta_{3}^{2}+{\sigma_{A 3}^{I}}^{I}\right)} \sim \delta_{1}^{3 / 2} .
\end{gathered}
$$

Amplitudes of the higher harmonics, $n>3$, can be found in the similar perturbative way, and one can see that their amplitudes are proportional to $\delta_{1}^{n / 2}$. Therefore, the harmonic series (11) converges and our perturbative approach is valid provided $\left|\delta_{1}\right| \ll 1$.

Thus in this section we have found the solution describing voltage oscillations in the vicinity of NDC region boundaries or for whole NDC region if it is small. Beyond the NDC region boundaries the oscillatory solution does not exist, this is reflected by the imaginary value of the oscillation amplitude in Eq. (14). Amplitude of the oscillations vanishes when the average voltage approaches NDC region boundary. Oscillation frequency is determined by geometry and by material properties of the sample. This solution exists provided the imaginary part of impedance of the effective antenna has the sign corresponding to inductance. 


\section{MICROWAVE RESPONSE}

In this section we determine modification of the I-V curve when the system is exposed to a weak external harmonic radiation of frequency $\omega$. Again, for simplicity, we treat antenna as an inductance. Let the incident radiation induces voltage oscillations with the amplitude $V_{A}$ at the antenna output. Then the equation for the oscillating component of the voltage (8) acquires the form

$$
\ddot{\theta}+\omega_{0}^{2} \theta+\frac{\delta \dot{\theta}+\frac{1}{2} \sigma_{d}^{\prime} \dot{\theta}^{2}+\frac{1}{6} \sigma_{d}^{\prime \prime} \dot{\theta}^{3}}{c_{s} s}=\frac{j-\left(\sigma_{1}+\sigma_{2}\right) v_{0} / s}{c_{s} s}+f \sin \omega \tau .
$$

Here $f=V_{A} \omega_{0}^{2} / \omega N s$ is the amplitude of the external force. This equation describes the forced oscillations of the system with single degree of freedom in case of periodic perturbation. As in the previous section we consider the case when the amplitude of the voltage oscillations is small and we solve equation for $\theta(\tau)$ perturbatively. When the frequency of the external field, $\omega$, is close to the eigen frequency, $\omega_{0}$, one can expect the effect of frequency capture. As in the previous section we consider the case when the amplitude of the voltage oscillations, $a$, is small. Then in the first approximation we seek the solution in the form of harmonic oscillations at the frequency of the external force but with unknown time-independent phase shift $\psi: \theta(\tau)=a \sin (\omega \tau+\psi)$. Then we find from Eq. (20)

$$
a \sqrt{A_{s}^{2}+A_{c}^{2}} \sin (\omega \tau+\psi+\varphi)=f \sin (\omega \tau)
$$

where $A_{s}=\omega_{0}^{2}-\omega^{2}, A_{c}=\omega\left(\delta+a^{2} \omega^{2} \sigma_{d}^{\prime \prime} / 8\right) / c_{s} s$, and $\tan \varphi=A_{c} / A_{s}$. Eq. (21) determines the amplitude of oscillations as function of frequency, $\omega$, and bias voltage, $v_{0}$. We seek solution of Eq. (21) for the amplitude in the form

$$
a=\frac{E_{1}}{\omega}(1+y)
$$

where the first term describes the voltage oscillations with the amplitude of the natural oscillations, $A_{1}$, given by Eq. (9). For small enough values of $f$ we find two solutions describing corrections to the oscillation amplitude induced by the external perturbation

$$
y\left(\omega, v_{0}\right)= \pm \frac{c_{s} s}{2 \omega \delta} \sqrt{\left(\frac{f \omega}{E_{1}}\right)^{2}-\left(\omega^{2}-\omega_{0}^{2}\right)^{2}}
$$

This expression is valid provided the external perturbation is small enough, $|f| \ll \delta^{3 / 2}$.

Corresponding expressions for the phase read

$$
\psi= \pm \arctan \frac{\sqrt{\left(f \omega / E_{1}\right)^{2}-\left(\omega^{2}-\omega_{0}^{2}\right)^{2}}}{\left|\omega^{2}-\omega_{0}^{2}\right|} .
$$

Using the approach of the next section one can show that both solutions found above are stable.

For $\omega=\omega_{0}$ the phases of the solutions are equal to $\pm \pi / 2$. In this case the first solution describes voltage oscillations in phase with the driving voltage, the oscillation amplitude being increased by the external perturbation, while the second solution corresponds to the out of phase oscillations with the amplitude decreased by the external force.

According to Eq. (22) the regime of frequency capture exists in the frequency range

$$
\left|\omega^{2}-\omega_{0}^{2}\right|<\frac{f \omega}{E_{1}}
$$

Variation of the I-V curve due to irradiation as function of frequency and DC bias is described by expression

$$
\Delta j=\frac{\sigma_{d}^{\prime} \omega^{2} E_{1}^{2}}{2} y
$$

Thus we can conclude that the external radiation induces two branches at the I-V curve that merge near the boundary of the regime of the frequency capture. Maximum variation of the amplitude and, hence, the largest shift of the I-V curve occur at $\omega=\omega_{0}$. They are linear in driving voltage.

At larger difference between the driving frequency and the eigen frequency, that is outside the regime of the frequency capture, corrections to the $\mathrm{I}-\mathrm{V}$ curve induced by the external perturbation are quadratic in $f$. 


\section{STABILITY}

Now we study the stability of the solution found in preceding section with respect to non-uniform perturbations of the voltage. Consider a sample consisting of $N$ layers. As it was stated above, we assume non-equilibrium processes to be slow enough in the scale of the energy relaxation time, so that distribution function of electrons inside conducting layers does not change. Then the in-layer electron distribution can be described by the Fermi distribution function with energy shifted by a non-equilibrium correction to the chemical potential induced by difference of the currents through adjacent conducting layers. At first we relate the shift of the chemical potential to the voltages in adjacent layers. Taking into account quasi-2D character of the layers, we obtain the expression for the difference of chemical potentials on the adjacent layers

$$
\delta \mu_{n}(\tau)=\frac{\hbar^{2}}{4 m e s}\left(V_{n}(\tau)-V_{n-1}(\tau)\right) .
$$

This allows us to express current in terms of voltages across different junctions only:

$$
\begin{aligned}
j_{n}^{(t)}(\tau)= & \frac{\sigma_{0}}{s}\left[\int_{0}^{\infty} d \tau_{1} e^{-\tau_{1}} \sin \int_{0}^{\tau 1}\left(V_{n}(\tau)-V_{n}\left(\tau-\tau_{2}\right)\right) d \tau_{2}\right. \\
& \left.-\frac{\partial_{n}^{2} V_{n}(\tau)}{4 m s} \int_{0}^{\infty} d \tau_{1} e^{-\tau_{1}} \cos \int_{0}^{\tau_{1}}\left(V_{n}(\tau)-V_{n}\left(\tau-\tau_{2}\right)\right) d \tau_{2}\right],
\end{aligned}
$$

where $\partial_{n}^{2} V_{n}=V_{n+1}+V_{n-1}-2 V_{n}$ is a discrete version of the second derivative on layer number.

Since sample dimensions are assumed to be small, the current in the external circuit is determined only by the total voltage across the sample:

$$
j^{(e x t)}(\tau)=\hat{\sigma}_{A}\left[\frac{1}{s N} \sum_{n} V_{n}(\tau)\right] .
$$

Using Eqs. (26) and (27) in Eq. (77) we obtain the equation describing voltage dynamics taking into account dependence of the voltage on the layer number:

$$
j_{n}^{(t)}+\sigma_{1} V_{n}(\tau)+\frac{1}{4 \pi s} \frac{\partial}{\partial \tau} V_{n}(\tau)=\hat{\sigma}_{A}\left[\frac{1}{s N} \sum_{k} V_{k}(\tau)\right] .
$$

We simplify Eq. (28) assuming that in the considered frequency range the antenna impedance can be modeled by pure inductance,

$$
\hat{\sigma}_{A}\left[\frac{1}{s N} \sum_{k} V_{k}(\tau)\right] \rightarrow \frac{1}{L S} \int \sum_{k} v_{k}(\tau) d \tau .
$$

Then each equation in Eq. (28) acquire a form of Eq. (8)

$$
\ddot{\theta}_{n}+\frac{\omega_{0}^{2}}{N} \sum_{k} \theta_{k}+\frac{1}{c_{s} s}\left(\delta \dot{\theta}_{n}+\frac{\sigma_{d}^{\prime} \dot{\theta}_{n}^{2}}{2}+\frac{\sigma_{d}^{\prime \prime} \dot{\theta}_{n}^{3}}{6}-j+\left(\sigma_{1}+N \sigma_{A}\right) \frac{v_{0}}{s}\right)=0 .
$$

To show that solution describing coherent voltage oscillations is stable, we substitute $\theta_{n}(\tau)=\theta^{(0)}(\tau)+\delta_{n}(\tau)$ into (29), where $\theta^{(0)}(\tau)$ is the solution found in Sec. IIII and then linearize the resulting equation in $\delta_{n}$. Temporal evolution of small perturbation $\delta_{n}(\tau)$ is described by equation:

$$
\ddot{\delta}_{n}+\left(\sigma(\tau)-D \partial_{n}^{2}\right) \dot{\delta}_{n}+\frac{\omega_{0}^{2}}{N} \sum_{k} \delta_{k}=0 .
$$

Here $\sigma(\tau)=\left(E_{1} \sigma_{d} \cos \left(\omega_{0} \tau+\varphi_{0}\right)+\frac{1}{8} \sigma_{d}^{\prime \prime} E_{1}^{2}\left(1+2 \cos 2\left(\omega_{0} \tau+\varphi_{0}\right)\right) / c_{s}\right.$ plays the role of time-dependent friction coefficient, and $D=\sigma_{0} / 4 m c_{s} s^{2}\left(1+v_{0}^{2}\right)$ is diffusion coefficient. 
The general solution of linear equations (30) can be divided into a uniform and a non-uniform parts: $\delta_{n}=\delta^{(0)}+\delta^{(n)}$, $n=1 . . N$. The uniform part of the solution, $\delta^{(0)}$, can be found as the general solution of the equation

$$
\ddot{\delta}^{(0)}(\tau)+\sigma(\tau) \dot{\delta}^{(0)}(\tau)+\omega_{0}^{2} \delta^{(0)}(\tau)=0,
$$

which follows from Eq. (30) when all $\delta_{n}$ are equal. The rest of Eq. (30) form $N$ identical equations for the non-uniform part of the perturbation, $\delta^{(n)}$

$$
\ddot{\delta}^{(n)}(\tau)+\left[\sigma(\tau)-D \partial_{n}^{2}\right] \dot{\delta}^{(n)}(\tau)=0,
$$

which satisfy the condition which ensures that the total voltage over all layers is described by $\delta^{(0)}$

$$
\sum_{n=1}^{N} \delta^{(n)}(\tau)=0 .
$$

So we examine the temporal evolution of the total voltage across the sample and voltage fluctuations at different junctions separately, by Eqs. (31) and (32), respectively.

At first we study non-uniform perturbations described by Eq. (32). Its solution can be found easily by means of the Fourier transformation on layer number:

$$
\delta_{k}(\tau)=c_{1}(k)+c_{2}(k) \int e^{-\int\left(\sigma(\tau)+D \hat{k}^{2}\right) d \tau} d \tau,
$$

where $\hat{k}=2 \sin k / 2$, and $c_{1}(k)$ and $c_{2}(k)$ are constants of integration. Constants $c_{1}(k)$ are irrelevant since they are not related to voltage fluctuations since voltage is determined by time derivative of the phase $\theta_{n}$, so we can equate these constants to zero. Since the diffusive term promotes the stability $(D>0)$, we concentrate on the case of small $\hat{k} \rightarrow 0$. Then the stability of the solution is determined by the damping term $\sigma(\tau)$ which can be presented as $\sigma(\tau)=\sigma_{0}+\sigma_{1}(\tau)$ where $\sigma_{1}(\tau)$ is a sum of harmonic terms, and the average value of the damping, $\sigma_{0}$, is positive. Since it is positive, Fourier transforms of the perturbation (34) decrease exponentially at $\tau \rightarrow \infty$ for all values of $k$.

Note that the results on stability with respect to non-uniform oscillations remain valid in the general case, when the external antenna cannot be described by pure inductance. Indeed, in case of antenna described by a linear operator of the total voltage across the sample, the uniform and non-uniform fluctuations can be examined separately similar to the way it was described above. Thus non-uniform perturbations cannot lead to instability of the oscillatory solution and the stability of the oscillatory regime is determined only by the stability of uniform oscillations.

Now we discuss the uniform perturbation. The general solution of (31) is

$$
\delta^{(0)}(\tau)=C_{1} v(\tau)+C_{2} v(\tau) \int \frac{1}{v^{2}(\tau)} e^{-\int \sigma(\tau) d \tau} d \tau .
$$

Here $C_{1}$ and $C_{2}$ are arbitrary constants. As, by definition, $\dot{\theta}^{(0)}(\tau)=v(\tau)$, the first term in (35) describes the solution equal to the unperturbed solution that is shifted by time $C_{1}$. This term is related to the different choice of initial conditions, and it is not related to stability. The second term decreases exponentially. This can be shown by direct calculations and demonstrated by means of the Ostrogradsky-Liouville formula relating the Wronskian to the coefficients of the linear differential equation,

$$
\left|\begin{array}{ll}
v(\tau) & \delta(\tau) \\
\dot{v}(\tau) & \dot{\delta}(\tau)
\end{array}\right|=C e^{-\int \sigma(\tau) d \tau}
$$

where $C$ is an arbitrary constant. With $\tau$ increase perturbed solution exponentially converges to non-perturbed one which may have some phase shift with respect to original solution, i.e., original uniform solution is stable.

\section{DISCUSSION}

In this section we discuss some aspects of experimental realization of the oscillatory regime. The condition for voltage oscillation derived in the previous section implies that the supercriticality must be negative, $\delta_{1}<0$. This is possible when additional conductivities due to non-coherent tunneling and antenna do not compensate the NDC due to coherent interlayer tunneling, $c f$. Eq. (6)

$$
\sigma_{1}+\sigma_{A 1}^{R}\left(\omega_{0}\right)<\sigma_{0} / 8
$$


Again we consider Eq. (8), in which we explicitly show the antenna impedance. As it was mentioned above, on main frequency the "friction" term vanishes, and the equation defining the frequency of the voltage oscillation reads

$$
\sigma_{0} \tau_{p} c_{s} \dot{v}+\frac{i}{S} \Im\left[Z_{A}^{-1}\right] v=0 .
$$

Since specific tunneling capacity $c_{s}$ does not change dramatically with $v_{0}$ changes, all conclusions remain valid for the case of boundaries of large NDC region either.

The oscillation frequency can be presented in the form

$$
\omega_{0}=\frac{1}{\tau_{p} c_{s} s} \Im \frac{R_{0}}{Z_{A}} .
$$

Here $R_{0}$ is resistance of the sample.

Note also, that from equation (11) for the tunneling current it follows that the oscillation frequency can not be large compared to the momentum scattering rate $1 / \tau_{p}$. This becomes clear, in particular, from Eq. (3) demonstrating that the differential conductivity becomes positive at high frequencies, $\omega>\tau_{p}^{-1}$.

According to Eq. (37) oscillation frequency depends on the input antenna impedance $Z_{A}$. Dependence $Z_{A}(\omega)$ can be very diverse for different antenna configurations, so to illustrate possibility of the proposed oscillatory regime we consider the most simple case of the dipole antenna consisting of thin straight wire of length $l_{A}$ and radius $r$. This antenna can be described by the long line theory 14 . Then antenna wave impedance in the SI units reads $\rho=\frac{1}{\pi c \varepsilon_{0}}\left(\ln \frac{2 l_{A}}{r}-1\right)[\Omega]=120\left(\ln \frac{2 l_{A}}{r}-1\right)[\Omega]$, where $k=2 \pi l_{A} / \lambda$, where $\lambda$ is the wave length. For the sending-end impedance this theory gives:

$$
Z_{A}=\frac{R_{A} \rho^{2}}{R_{A}^{2}+\rho^{2} \sin ^{2} k l_{a}}-i \frac{\rho^{3} \sin 2 k l_{A}}{2\left(R_{A}^{2}+\rho^{2} \sin ^{2} k l_{A}\right)}
$$

Here, the dipole resistance $R_{A}$ is a sum of the radiation resistance related to the antinode of the current, and of the ohmic resistance of the antenna's material, the latter is assumed to be much smaller than the former one. The radiation resistance is given by expression $R_{\Sigma}=P / I^{2}=30\left(2\left(\gamma+\ln 2 k l_{A}-\mathrm{Ci} 2 k l_{A}\right)+\left(\gamma+\ln k l_{A}+\mathrm{Ci} 4 k l_{A}-2 \mathrm{Ci} 2 k l_{A}\right) \cos 2 k l_{A}+\right.$ $\left.\left(\mathrm{Si} 4 k l_{A}-2 \mathrm{Ci} 2 k l_{A}\right) \sin 2 k l_{A}\right)[\Omega]$ (here $P$ is radiation power, $\gamma$ is Euler constant, $\mathrm{Ci}$ and $\mathrm{Si}$ are integral cosine and sine, respectively).

According to Eq. (38) the imaginary part of the antenna impedance is oscillatory function of frequency and on the length of the antenna arms $l_{A}$. The dependence of real and imaginary parts of $Z_{A}^{-1}$ on $l_{A}$ is presented in Fig. 3 for several sets of antenna parameters. When the ratio $l_{A} / \lambda$ is in the range from 0.5 to $1, \Im Z_{A}$ is positive and the oscillatory solution is possible.

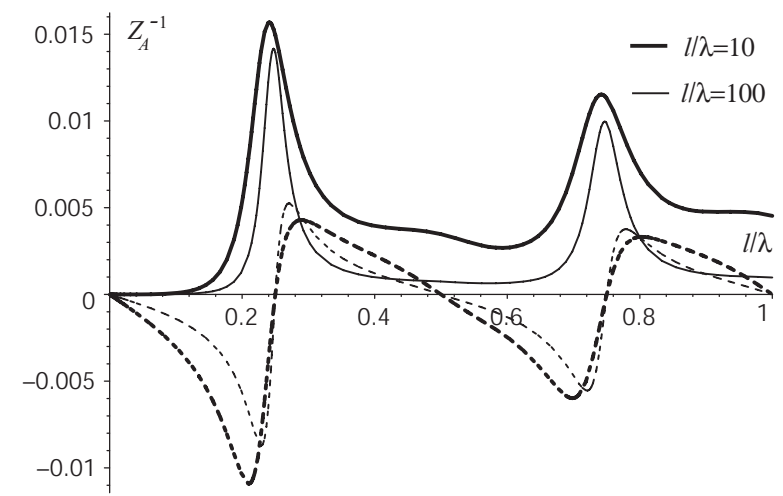

FIG. 3: Dependence of $Z_{A}^{-1}$ on ratio $l_{A} / \lambda$ for simple case of dipole antenna. Solid and dashed lines correspond to active resistance and reactive impedance, respectively.

To estimate resistivity of the sample, we use conductivity data for $\mathrm{NbSe}_{3}$ adduced in Ref 7 for $\mathrm{NbSe}_{3}, \sigma_{0}=$ $0.1 \mathrm{~m} \Omega \mathrm{cm}$, and assume the sample dimensions to be $10 \mu \mathrm{m} \times 10 \mu \mathrm{m} \times 300 \AA$ with the smallest length in the current direction. Then we obtain $R_{0}=30 \Omega$. Typical values of $\Re Z_{A}$ for the most interesting case of antenna with relatively large wave impedance which corresponds to high Q-value, are of the order of several $k \Omega$ (see Fig. B), hence, the antenna impedance is larger then the sample resistance $\left(R_{0} \Im Z_{A}^{-1}<1\right)$. So conductivities $\sigma_{A n}^{I}$ and $\sigma_{A n}^{R}$ are small, as it was supposed in previous sections. 
For practical applications the higher frequencies are more interesting. We see several possible ways to increase the oscillation frequency. The first one is to increase $R_{0}$ by increasing the ratio $s N / S$, either by changing a sample geometry or by increasing the number of layers. Implementation of this idea is limited by the condition for the NDC region to exist (36) due to an increase of relative dissipation by antenna caused by radiation as $\sigma_{A 1}^{R} \propto s N / S$. Another way is to maximize $\Im Z_{A}^{-1}$ in order to optimize the ratio of the antenna arm length and the wavelength, or to use more complicated antenna, than the simple dipole one. In this case the limitations are imposed by real part of antenna impedance related to radiation power $R_{\Sigma}$ - practically interesting case of larger radiation leads to larger $R_{\Sigma}$ while $\Im Z_{A}^{-1}<R_{\Sigma}^{-1} / 2$.

In previous sections we assumed that the current density does not depend on the in-plane coordinate. In addition to the non-uniformity related to fluctuations of charge density considered in Sec. V there is another non-uniformity which is related to the skin effect. So, strictly speaking, our results are applicable provided the sample width is smaller, than the skin-effect length, $c / \sqrt{2 \pi\left(\sigma_{1}+\sigma_{0} /\left(1+v_{0}^{2}\right)\right) \omega_{0}}$. This condition can be easily satisfied in the tera-Herz region.

In conclusion, we have studied the problem of the current flow through the sample with narrow energy band when the average voltage corresponds to the NDC region. We have found analytically the stable uniform oscillating solution describing coherent voltage oscillations in the sample. The amplitude and the frequency of the oscillations are determined by dimensions, geometry, and material properties of the sample, and depend on the antenna properties of the system. The maximum value of the oscillation frequency is limited by the momentum relaxation rate.

\section{ACKNOWLEDGMENTS}

We are grateful to V.A. Volkov for helpful comments and to V.Ya. Aleshkin for useful information. In part the work was supported by Russian Foundation for Basic Research (RFBR), by INTAS, and by CRDF. A part of these research was performed in the frame of the CNRS-RAS-RFBR Associated European Laboratory "Physical properties of coherent electronic states in condensed matter" between CRTBT and IRE RAS.

1 R. L. Withers and J. A. Wilson, J. Phys. C 19, 4809 (1986).

2 R. Kleiner and P. Müller, Phys. Rev. B 49, 1327 (1994). R. Kleiner, P. Müller, H. Kohlstedt, et al., Phys. Rev. B 50, 3942 (1994).

3 N.P. Ong and J.W. Brill, Phys. Rev. B 18, 5265 (1978); Yu.I. Latyshev, P. Monceau, O.Laborde, et al., J. Phys. IV 9, 165 (1999).

4 Andreas Wacker "Semiconductor superlattices: a model system for nonlinear transport", Physics Reports 357, pp. 1-111 (2002).

${ }^{5}$ L. Esaki and R. Tsu, IBM J. Res. Dev., vol. 14, pp. 61-65 (1970); L. Esaki and R. Tsu, Appl. Phys. Lett, 19, 246 (1971).

${ }^{6}$ S.N. Artemenko and A.F. Volkov, Fiz. Tverd. Tela (Leningrad) 23, 2153 (1981); Sov. Phys. Solid State 23, 1257 (1981)

7 Yu.I. Latyshev, A.A. Sinchenko, L.N. Bulaevskii, V.N. Pavlenko, P. Monceau, JETP Letters, 75, 93 (2002)

8 M.I. Flik, Z.M. Zhang, K.E. Goodson, M.P. Siegal and Julia M. Phillips, Phys. Rev. B 46, 5606-5614 (1992); D. A. Bonn, Ruixing Liang, T. M. Riseman, D. J. Baar, D. C. Morgan, Kuan Zhang, P. Dosanjh, T. L. Duty, A. MacFarlane, G. D. Morris, J. H. Brewer, W. N. Hardy, C. Kallin and A. J. Berlinsky, Phys. Rev. B 47, 11314-11328 (1993); F. Gao, J. W. Kruse, C. E. Platt, M. Feng, and M. V. Klein, Appl. Phys. Lett., 63, pp. 2274-2276 (1993).

9 H. Kroemer, cond-mat/0007482 (2000); cond-mat/0009311 (2000); Yu. A. Romanov, V.P. Bovin, and L. K. Orlov, Fiz. Tekh. Poluprovodn. 12, 1665 (1978) [Sov. Phys. Semicond. 12, 987 (1978)]

10 Yu.A. Romanov and Yu.Yu. Romanova, Semiconductors, 39, 147 (2005).

11 Yu.A. Romanov and Yu.Yu. Romanova, Physics of the Solid State, 46, 164 (2004).

12 E.M. Lifshits and L.P. Pitaevskii, Physical kinetics, (Pergamon Press, London, 1981).

13 I.O. Kulik and I.K. Yanson, Josephson Effect in Superconducting Tunnel Structures, Nauka, Moscow, (1970).

14 Sazonov D.M., Antennas and Microwaves Devices, High School(USSR), 1988.-432 p., English translation: Microwave Circuits and Antennas, Mir Publishers, Moscow, 1990, 504 p. 